University of Nebraska - Lincoln

DigitalCommons@University of Nebraska - Lincoln

USDA National Wildlife Research Center - Staff Publications
U.S. Department of Agriculture: Animal and Plant Health Inspection Service

2010

\title{
Comparison of Endocrine Response to Stress Between Captive- Raised and Wild-Caught Bighorn Sheep
}

\author{
Sarah Coburn \\ Colorado State University \\ Mo Salman \\ Colorado State University, Animal Population Health Institute \\ Jack Rhyan \\ USDA-APHIS-Wildlife Services \\ Thomas Keefe \\ Colorado State University - Fort Collins \\ Matthew McCollum \\ USDA-APHIS-Wildlife Services
}

See next page for additional authors

Follow this and additional works at: https://digitalcommons.unl.edu/icwdm_usdanwrc

Part of the Environmental Sciences Commons

Coburn, Sarah; Salman, Mo; Rhyan, Jack; Keefe, Thomas; McCollum, Matthew; Aune, Keith; Spraker, Terry; and Miller, Lowell, "Comparison of Endocrine Response to Stress Between Captive-Raised and WildCaught Bighorn Sheep" (2010). USDA National Wildlife Research Center - Staff Publications. 876.

https://digitalcommons.unl.edu/icwdm_usdanwrc/876

This Article is brought to you for free and open access by the U.S. Department of Agriculture: Animal and Plant Health Inspection Service at DigitalCommons@University of Nebraska - Lincoln. It has been accepted for inclusion in USDA National Wildlife Research Center - Staff Publications by an authorized administrator of DigitalCommons@University of Nebraska - Lincoln. 


\section{Authors}

Sarah Coburn, Mo Salman, Jack Rhyan, Thomas Keefe, Matthew McCollum, Keith Aune, Terry Spraker, and Lowell Miller 


\title{
Comparison of Endocrine Response to Stress Between Captive-Raised and Wild-Caught Bighorn Sheep
}

SARAH COBURN, Colorado State University, Animal Population Health Institute, College of Veterinary Medicine and Biomedical Sciences, Fort Collins, CO 80523, USA

MO SALMAN, ${ }^{1}$ Colorado State University, Animal Population Health Institute, College of Veterinary Medicine and Biomedical Sciences, Fort Collins, CO 80523, USA

JACK RHYAN, United States Department of Agriculture National Wildlife Research Center, 4101 LaPorte Avenue, Fort Collins, CO 80521, USA

THOMAS KEEFE, Colorado State University, College of Veterinary Medicine and Biomedical Sciences, Fort Collins, CO 80523, USA

MATTHEW MCCOLLUM, United States Department of Agriculture National Wildlife Research Center, 4101 LaPorte Avenue, Fort Collins, CO 80521, USA

KEITH AUNE, Montana Fish, Wildlife, and Parks, P.O. Box 200701, 1420 ED 6th Avenue, Helena, MT 59620, USA

TERRY SPRAKER, Colorado State University, College of Veterinary Medicine and Biomedical Sciences, Fort Collins, CO 80523, USA

LOWELL MILLER, United States Department of Agriculture National Wildlife Research Center, 4101 LaPorte Avenue, Fort Collins, CO 80521, USA

\begin{abstract}
Stress hormones in Rocky Mountain bighorn sheep (Ovis canadensis canadensis), produced in response to environmental changes, road development, or high population density, may impact their immune systems to a threshold level that predisposes them to periodic, large-scale mortality. We compared the stress response to a novel environmental situation and repeated handling between bighorn sheep born and raised in captivity (CR) and bighorn sheep born in the wild (WC) and brought into captivity. We measured plasma epinephrine, norepinephrine, cortisol, and fecal glucocorticoid metabolites (FGM). Three weeks after each group's arrival we used a one-time drop-net event to elicit an acute stress response, and we collected blood samples from each sheep over 35 minutes, as well as one fecal sample. We collected blood and fecal samples from both groups on 7 other occasions over the subsequent 6 months. We also collected fecal samples from the pen at approximately 24-hour intervals for 3 days following every handling event to monitor the stress response to handling. We found that CR sheep had a stronger autonomic nervous system response than WC sheep, as measured by epinephrine and norepinephrine levels, but we found a very similar hypothalamic-pituitary-adrenal axis (HPA) response, measured by cortisol levels, to the acute stress event of a drop-net restraint. We also found that once the WC sheep had acclimated, as indicated by the return to the initial baseline FGM levels within 12 weeks, the CR and WC groups' HPA responses to sampling events were not significantly different from one another. Fecal samples can provide a noninvasive mechanism for managers to monitor baseline FGM for a given herd. Using long-term monitoring of FGM rather than values from a single point in time may allow managers to correlate these levels to outside influences on the herd and better understand the impacts of management changes, population density, or increased human developments on the health of the sheep population.
\end{abstract}

KEY WORDS bighorn sheep, catecholamines, fecal glucocorticoids, measuring responses, Ovis canadensis, stress measurement.

Rocky Mountain bighorn sheep (Ovis canadensis canadensis; hereafter sheep) populations in the western United States experienced severe declines during the late 1800s (Buechner 1960). Periodic large-scale disease outbreaks and subsequent fatalities in the remaining herds continue to challenge managers (Silflow et al. 1989, Singer and Gudorf 1999, Monello et al. 2001). Human recreational pursuits, such as snowmobiling, may influence wildlife populations (Creel et al. 2002, Arlettaz et al. 2007). Sheep herds have been successfully reintroduced to some historic ranges, but many translocation efforts have failed because of disease outbreaks (Bailey and Klein 1997, Singer and Gudorf 1999). Pneumonia reportedly caused many of the die-offs, but mortalities are attributed to a combination of several different factors, and no one etiology has been identified in common to all of the mortality events (Forrester 1971, Festa-Bianchet 1988). More recently, researchers have hypothesized that stress influences susceptibility of sheep to certain pathogens because many types of stressors have been shown to suppress immune function (Belden et al. 1990, Miller et al. 1991, Ullrey 1993). With any

${ }^{1}$ E-mail: m.d.salman@colostate.edu translocation and reintroduction effort, animals are exposed to multiple stressors including environmental disturbance, capture, captivity, veterinary examinations, and transport, the combination of which may ultimately affect the success of the reintroduction (Teixeira et al. 2007).

Responses to stress may be acute or chronic. Acute stress occurs when short-lived environmental factors require some immediate behavioral or short-term physiological response from the animal to avoid a negative outcome (Moberg 2000). Conversely, chronic stress is a condition caused by one stressor or a series of stress events of sufficient magnitude and duration that require resources to be shifted from other biological functions, such as metabolism, growth, reproduction, or immune function, in order to cope with the stressor (Moberg 2000). Chronic stress may be an important phenomenon leading to long-term physiological changes and susceptibility to disease (Ladewig 2000, de Kloet 2004). Susceptibility of sheep to pathogens may be, in part, caused by nutritional or disturbance-related stress that subsequently leads to suppression of the immune system (Spraker et al. 1984). Glucocorticoid production in the body, primarily cortisol in mammals, increases in response to acute stressors, such as transport or restraint, 
and can be used to monitor this stress response (Möstl et al. 2002).

Circulating plasma cortisol can be measured, but collecting the sample often elicits a stress response and confounds the results. Therefore, researchers are increasingly using the noninvasive collection of fecal samples in a variety of species to detect endocrine hormones released in response to stress (Miller et al. 1991, Wasser et al. 2000, Millspaugh et al. 2002). In domestic sheep approximately $28 \%$ of radiolabeled cortisol metabolites were excreted in the feces (Möstl et al. 2002).

One radioimmunoassay kit designed to measure corticosterone in rat plasma is commercially available and has been widely used in a variety of species to monitor fecal glucocorticoid metabolites (FGM; Creel et al. 1997, Wasser et al. 2000, Millspaugh et al. 2001, Li et al. 2007). Researchers have identified 21 glucocorticoid metabolites using high-performance liquid chromatography and mass spectrometry, but only trace amounts of native cortisol or corticosterone have been identified in fecal samples from domestic sheep; hence, we use the term fecal glucocorticoid metabolites to encompass any of the 21 different metabolites that researchers have recovered (Palme et al. 1999). When the rat corticosterone kit is used on species other than rats and for fecal cortisol metabolites rather than plasma, the specific metabolites measured are not known, except to say that they are not cortisol or corticosterone. Therefore, the absolute level of FGM should be used with caution, and specific values from different studies should not be compared (Millspaugh and Washburn 2004). However, overall trends in FGM levels may provide valuable information and allow for more valid comparisons among studies (Millspaugh and Washburn 2004). Group-specific assays have been developed, and their use in future research may provide an even better understanding of the biological relevance of specific FGM in animals (Morrow et al. 2002, Palme 2005).

Catecholamines, such as epinephrine and norepinephrine, represent the activation of the autonomic nervous system (ANS) and are released when an animal is actively responding to a particular threat or stressor (Henry 1993). Corticosteroids represent stimulation of the hypothalamicpituitary-adrenal axis (HPA), which is preferentially activated when an animal perceives that it has no control over the situation and does not attempt to fight back (Corley et al. 1975, de Boer et al. 1990). The perception by an animal that it has no control over a stressor has been referred to as learned helplessness (Seligman and Maier 1967). The impact of stress on animal health has been shown to be more severe in situations where the animal perceives that it is unable to predict, prevent, or otherwise control the stressors it is facing (Corley et al. 1975). Chronic psychological stress has been simulated in laboratory environments by exposing animals to an unpredictable schedule of acute stressors such as loud noises or exposure to predator decoys (Cyr and Romero 2007). Given the complexity of the physiological relationships between stress and disease and the current lack of reliable mechanisms to measure chronic stress, a combination of measurements of the HPA and ANS may provide a more complete overall picture of stress and animal well-being.

Our objectives were to compare and contrast the HPA and ANS responses to an acute drop-net stress event (a large, square net was remotely dropped over the animals to allow for immediate blood sample collection) and to repeated routine handling over time, between wild sheep brought into captivity (WC) and sheep raised in captivity (CR). We sought to use the changes in FGM levels following an acute stress event over time as an index of the chronic stress the sheep experienced. We hypothesized that the CR sheep would quickly acclimate to their new environment, as determined by lower level increases in their FGM in response to the acute stress of handling, as compared to WC sheep that would have higher levels of FGM following handling than the CR. We also hypothesized that plasma epinephrine, norepinephrine, and cortisol would increase more quickly and peak higher in $\mathrm{WC}$ than $\mathrm{CR}$ sheep in response to the drop-net event.

\section{STUDY AREA}

The Animal Population Health Institute (APHI) animal facility at Colorado State University in Fort Collins, Colorado, USA, contained $30-\mathrm{m} \times 30-\mathrm{m}$ pens to house each group of sheep. Each pen had a gate on the outer perimeter that led to a 4-m-wide $\times 85$-m-long alley. The alley connected to the chute handling area. This $15-\mathrm{m} \times$ 10- $\mathrm{m}$ area contained a concrete foundation with a squeeze chute designed for restraint and handling of deer (Delclayna Mfg, Swanville, MN). The animals had free-choice access to hay and water. We placed large rock piles in each pen for enrichment. Three-sided sheds were available for shelter.

\section{METHODS}

In the spring of 2004, we transported 2 groups of sheep to the APHI animal facility in Fort Collins, Colorado. The first group consisted of $20 \mathrm{CR}$ animals (10 ad $\mathrm{M}$ and $10 \mathrm{ad}$ $\mathrm{F}$ all $>2 \mathrm{yr}$ old). These sheep were raised in 3 source herds: 1) Wildlife Health Laboratory in Caldwell, Idaho, USA (WHL); 2) Sybille Wildlife Research Center and Wyoming Game and Fish Department in Wheatland, Wyoming, USA; and 3) Colorado Division of Wildlife in Fort Collins, Colorado. Prior to our study all CR sheep were housed at the WHL. The second group consisted of $14 \mathrm{WC}$ animals ( 9 ad $F$ and 2 ad $M>2$ yr old; 2 yearling $F$ and 1 yearling $\mathrm{M})$, which we captured by helicopter net-gun in Montana, USA, and immediately transported to the study facility. We visually monitored and recorded general observations of each animal at least twice daily, when animals were feeding. On most days the animals were observed more frequently while researchers and animal health technicians were working at the facility. Veterinarians were on-site or available by phone at all times. We conducted animal care and handling according to the guidelines established by the Animal Care and Use Committee of Colorado State University (Institutional Animal Care and Use Committee project no. 04-034A-01). 
We used the squeeze chute for periodic handling of the sheep and blood- and fecal-sample collection. To move the sheep from the pen to the chute, 3 animal handlers walked into the pen and used their movement within the flight zone of the sheep to push them into the alley. The flight zone is the area around an animal that when encroached upon will cause the animal to move in response. Once the sheep were in the alley, the animal handlers continued to walk behind the sheep to move them into a smaller holding pen, which funneled into a long, enclosed tunnel that led into the squeeze chute. After the initial contact with animal handlers in the pen, the first sheep would be in the chute within 515 minutes. Sample collection required 3-10 minutes per sheep. Approximately 1 hour was required to move the sheep to the handling area and collect samples from each sheep in the group. Between 6 and 8 animal handlers were available to move the sheep, collect samples, work the chute, and record data during each handling event.

We used capture by drop-net to elicit an acute stress response approximately 3 weeks after each group of sheep arrived at the APHI facility (15 Mar 2004 for WC; 3 Mar 2004 for CR). We set up the net the night before the sampling event to minimize human noise and activity on the morning of the sampling. We used hay and grain to lure the sheep under the net, which we then dropped by remote control. After we dropped the net, a group of 3 animal handlers per sheep immediately restrained, blind-folded, and removed each sheep from beneath the net. We restrained each animal with hobbles (nylon straps used to tether the legs together) for 35 minutes. During this time we collected blood samples at approximately 5-minute intervals by jugular venipuncture, and we placed samples in 7-mL ethylene diamine tetraacetic acid (EDTA) blood collection tubes (BD Vacutainer, Franklin Lakes, NJ). We recorded the times of the net-dropping and of each subsequent blood sample collection. This sampling method allowed for comparison of the immediate stress responses between the 2 groups to an acute stress event.

We collected the first fecal sample from WC sheep at the time of capture (27 Feb 2004), and we collected the first sample for CR sheep 10 days after transport to the study facility (17 Feb 2004). We collected fecal samples from the rectum of each animal when we handled them under the drop-net and in the chute. We collected samples 8 times between 17 February 2004 and 27 July 2004. We reported samples that we collected rectally during handling under collection day 1 , and we reported samples collected from the pens on the following days according to collection days 2, 3, or 4. To account for diurnal variation in cortisol metabolite levels, we collected samples between 0600 hours and 1000 hours. We attempted to collect fecal samples from each sheep once a day for 3 consecutive days subsequent to a handling event. When possible, we identified the specific animal associated with each fecal sample.

To collect samples from the WC sheep, we moved the herd into another paddock (clean of fecal material) for approximately 30 minutes and then returned it to its pen. We collected samples from each of the fecal piles and assumed that each pile was from a different sheep. We collected fecal samples from the CR sheep by observing them lying down early in the morning. When they first stood up and defecated, we collected the sample and recorded the animal identification number. We collected samples in individual Whirl-Paks (Nasco, Fort Atkinson, WI) and stored them at $-80^{\circ} \mathrm{C}$ until processed. We categorized samples by sampling event (1-8) and day (1-4) within the sampling period. A subset of the samples collected each day was selected for analysis.

We defined the baseline as the background FGM level prior to the stress of a handling event, in our study the FGM on day 1. By measuring the baseline level and the subsequent increase in FGM following these acute stress events over time, we made a general assessment of the chronic stress that the sheep may have been experiencing. We assumed FGM present in a given fecal sample represented the glucocorticoids that were produced approximately 12 hours previously. For the WC sheep, fecal samples collected at the time of capture in Montana represented FGM prior to handling and transport and we assumed they were the baseline level for this group. By collecting fecal samples for 3 days following each handling event, we were able to monitor the response to an acute stressor.

To extract FGM, we thawed the frozen samples and blended them to a fine-particle consistency. We vortexed $1 \mathrm{~g}$ of feces (wet wt) for 21-minute intervals in $5 \mathrm{~mL}$ of $80 \%$ methanol, with 1 minute of rest in between. We then centrifuged the feces-methanol mixture for 15 minutes at $2,500 \mathrm{rpm}$. We removed the supernatant and stored it at $-80^{\circ} \mathrm{C}$. We randomly selected a subset from each sample period, collection day, and group for processing and analysis.

We used commercially available radioimmunoassay kits from MP Biomedicals (Costa Mesa, CA) to measure cortisol (Catalog no. 06B-256440), epinephrine, and norepinephrine (Catalog no. 07L-114102) in EDTA plasma samples, according to manufacturer's instructions. We determined FGM levels using a double-antibody, rat corticosterone kit (MP Biomedicals Catalog no. 07120103). To adapt the assay, which was designed for serum, to the FGM levels in these fecal samples, we used a 1:5 dilution with the steroid diluent provided in the kit for all FGM assays. We based this procedure upon the standard curve acquired from the radioimmunoassay kit and the amounts of glucocorticoids determined from our samples.

We considered each analysis statistically significant if its probability value ( $P$-value) was $\leq 0.05$. We divided blood samples from the drop-net sampling event into 75 -minute time periods according to the number of minutes post-dropnet that we collected the sample. We used analysis of variance (ANOVA) for repeated measures to compare average plasma hormone levels (cortisol, epinephrine, and norepinephrine) between the $\mathrm{CR}$ and $\mathrm{WC}$ groups at each time period and among the 7 time periods following the drop-net. We conducted our study in concert with another study that required euthanasia of some of the animals over the course of the 6 months. Therefore, not all animals in our 


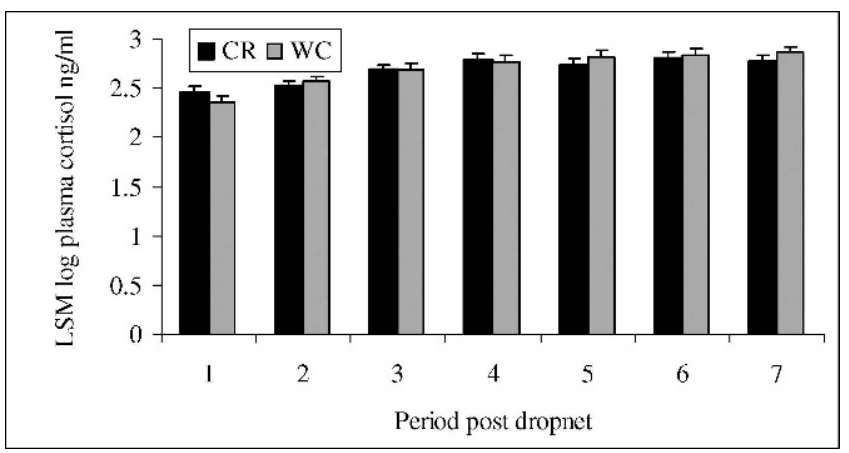

Figure 1. Plasma cortisol levels in bighorn sheep following a drop-net event at the Animal Population Health Institute, Colorado State University animal facility, Fort Collins, Colorado, USA, 3 March and 15 March 2004. Base-10 log-transformed least squares means (LSM) for plasma cortisol levels in captive-raised (CR) and wild-caught (WC) bighorn sheep during 7 time periods of 5-minute intervals following a drop-net sampling event are shown.

study were available for all sampling periods. We used leastsquares means (LSM) to account for differences in the number of samples collected within each time period. We used the Statistical Analysis Software Program PROC MIXED to complete all analyses (SAS Institute, Cary, NC). We also used repeated measures ANOVA to compare average FGM levels between the 2 groups (CR and WC) at day 1 of each sampling period (sample periods 1-8) and among the 4 collection days (days 1-4). To meet the assumptions of homogeneity of variance and normality, we applied a base-10 log transformation to drop-net cortisol, epinephrine, and norepinephrine values in plasma, as well as to FGM values. The $\mathrm{WC}$ and $\mathrm{CR}$ groups were not balanced according to age and sex; therefore, we did not include these parameters in our statistical analysis.

\section{RESULTS}

During the drop-net events, 54 blood samples were collected from 11 different CR animals, and 72 blood samples were collected from 12 different WC sheep. We report drop-net sample results according to time period post-drop-net during which we collected samples. Plasma cortisol levels that we obtained during the drop-net sampling ranged from $1 \mathrm{ng} / \mathrm{mL}$ to $7.5 \mathrm{ng} / \mathrm{mL}$ for the $\mathrm{CR}$ sheep and $1.1 \mathrm{ng} / \mathrm{mL}$ to $15.0 \mathrm{ng} / \mathrm{mL}$ for the WC sheep. Overall, plasma cortisol levels for both groups steadily increased throughout the 75 -minute sampling occasions (35 min; Fig. 1). We compared the LSM of log-transformed plasma cortisol levels, but we did not detect any statistically significant differences in plasma cortisol between $\mathrm{CR}$ and WC groups at any of the 7 time periods (CR LSM ranged from 2.45 to 2.80 and $\mathrm{WC}$ from 2.35 to $2.86, P-$ values ranged from 0.32 to $0.97, \mathrm{df}=82$ ).

Epinephrine levels for the $\mathrm{CR}$ group ranged from 7.0 $\mathrm{ng} / \mathrm{mL}$ to $185.5 \mathrm{ng} / \mathrm{mL}$. Values for the $\mathrm{WC}$ group ranged from $0.01 \mathrm{ng} / \mathrm{mL}$ to $70.5 \mathrm{ng} / \mathrm{mL}$. We compared the LSM of log-transformed plasma epinephrine levels between the $\mathrm{CR}$ and WC groups among 7 post-drop-net time periods. Overall epinephrine levels in CR were significantly higher than levels in WC sheep $(\mathrm{CR}$ LSM $=2.08, \mathrm{WC}$ LSM $=$

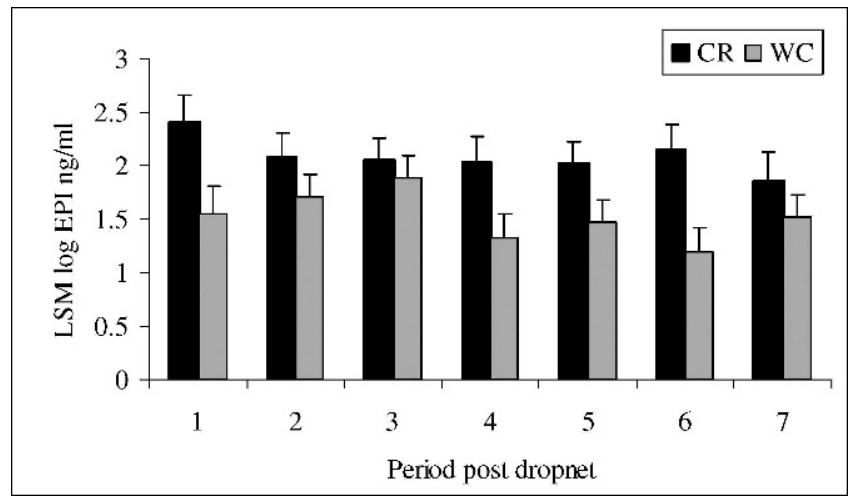

Figure 2. Plasma epinephrine (EPI) levels in bighorn sheep following a drop-net event at the Animal Population Health Institute, Colorado State University animal facility, Fort Collins, Colorado, USA, 3 March and 15 March 2004. Base-10 log-transformed least squares means (LSM) for plasma EPI levels for captive-raised (CR) and wild-caught (WC) bighorn sheep during 7 time periods of 5-minute intervals following a drop-net sampling event are shown.

1.52, $P=0.025$, $\mathrm{df}=19)$. Captive-raised values were numerically higher at each time period, but the mean difference between the $\mathrm{CR}$ and $\mathrm{WC}$ groups was statistically significant only at time periods $1(\mathrm{CR}$ LSM $=2.41, \mathrm{WC}$ $\mathrm{LSM}=1.55, P=0.021, \mathrm{df}=82), 4(\mathrm{CR} \mathrm{LSM}=2.04$, $\mathrm{WC} \operatorname{LSM}=1.31, P=0.034)$, and $6(\mathrm{CR} \mathrm{LSM}=2.14$, $\mathrm{WC} \operatorname{LSM}=1.20, P=0.01$; Fig. 2$)$.

The CR plasma norepinephrine levels ranged from 0.3 $\mathrm{ng} / \mathrm{mL}$ to $100.1 \mathrm{ng} / \mathrm{mL}$. The $\mathrm{WC}$ norepinephrine values ranged from $0.01 \mathrm{ng} / \mathrm{mL}$ to $22.7 \mathrm{ng} / \mathrm{mL}$. The only statistically significant difference between the 2 groups occurred at time period 6 with the LSM being significantly higher for the CR group than for the WC group (CR LSM $=1.41, \mathrm{WC} \operatorname{LSM}=0.60, P \leq 0.001, \mathrm{df}=82)$. Overall, there was no significant difference in norepinephrine levels between CR and WC (CR LSM $=1.12$, WC LSM $=1.04$, $P=0.54$, df $=19$; Fig. 3 ).

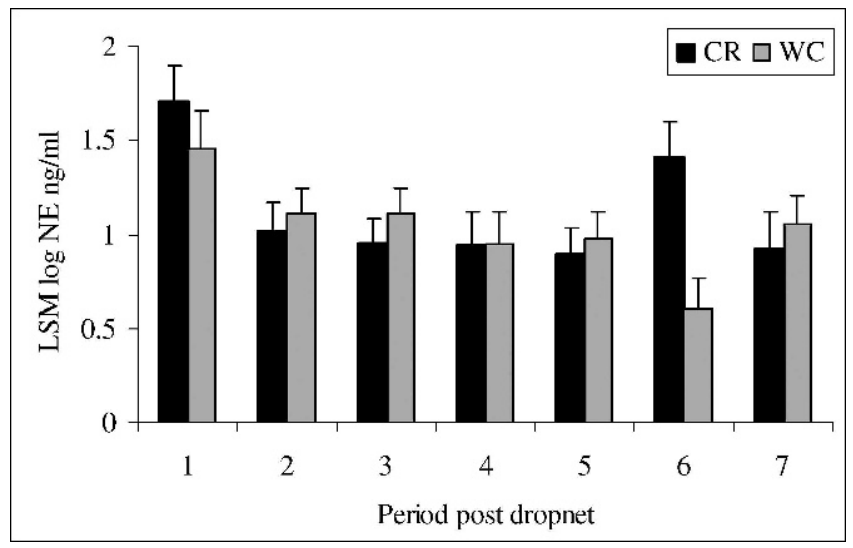

Figure 3. Plasma norepinephrine (NE) levels in bighorn sheep following a drop-net event at the Animal Population Health Institute, Colorado State University animal facility, Fort Collins, Colorado, USA, 3 March and 15 March 2004. Base-10 log-transformed least squares means (LSM) for plasma NE levels for captive-raised (CR) and wild-caught (WC) bighorn sheep during 7 time periods of 5-minute intervals following a drop-net sampling event are shown. 


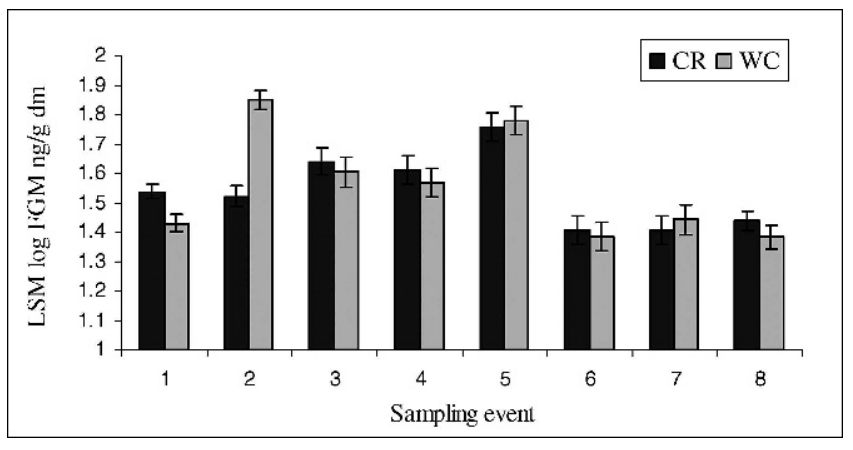

Figure 4. Changes in baseline fecal glucocorticoid metabolites (FGM) collected February-July 2004 from bighorn sheep relocated to the Animal Population Health Institute, Colorado State University animal facility, Fort Collins, Colorado, USA, February-July 2004. Base-10 log-transformed least squares means (LSM) for FGM in captive-raised (CR) and wildcaught (WC) bighorn sheep are shown. We handled animals 8 times over 6 months. Sampling events occurred at intervals of 2-4 weeks.

We collected approximately 1,200 fecal samples total, and analyzed FGM in 475 . We report results by sample event (1-8) and by collection day (1-4) within each event for the $\mathrm{CR}$ and WC groups. We compared least squares means of log-transformed FGM levels for CR and WC on collection day 1 among sampling events $1-8$.

For the WC group, the mean FGM for collection day 1 in sample event 1 (LSM = 1.43), collected at the time of capture, were statistically lower than those at sample events $2(\mathrm{LSM}=1.85, P \leq 0.001 ; \mathrm{df}=156), 3(\mathrm{LSM}=1.60, P$ $=0.003), 4(\mathrm{LSM}=1.57, P=0.017)$, and $5(\mathrm{LSM}=1.78$, $P<0.0001)$ but were not statistically different from mean FGM at sample events $6(\mathrm{LSM}=1.39, P=0.44), 7(\mathrm{LSM}$ $=1.44, P=0.44)$, and 8 (LSM $=1.38, P=0.35$; Fig. 4$)$. Mean FGM values for collection day 1 in sample events 2 and 5 were significantly higher than mean FGM for all other sample events in day 1 (all $P$-values $\leq 0.001$ ).

For CR sheep, FGM levels for collection day 1 obtained during sample event $5(\mathrm{LSM}=1.76$, df $=156)$ were significantly higher than those from time periods 1 (LSM $=$ 1.54), $2(\mathrm{LSM}=1.52), 4(\mathrm{LSM}=1.61), 6(\mathrm{LSM}=1.41)$, $7(\mathrm{LSM}=1.41)$, and $8(\mathrm{LSM}=1.44$; all $P$-values $\leq 0.001$, $\mathrm{df}=156)$. Similar to the WC sheep, FGM values for collection day 1 for sample events 1 through 5 were higher than those at events 6, 7, and 8. Collection day 1 in sample events 1, $3(\mathrm{LSM}=1.64), 4$, and 5 were all significantly higher than 6, 7, and 8 in mean FGM $(P<0.05)$.

Levels of FGM for collection day 1 for WC sheep were significantly lower than $\mathrm{CR}$ values at sampling event $1(P=$ $0.006)$ and higher than $\mathrm{CR}$ value at sampling event $2(P \leq$ 0.001). Mean differences in FGM for collection day 1 between the groups were not statistically significant at any other sampling events. For WC sheep the FGM levels returned to baseline after approximately 12 weeks.

There were no overall differences in FGM levels between the CR and WC groups among sampling events 3-8 when we only considered collection day 1 levels; however, there were significant differences in what collection day (1-4) the FGM levels peaked following handling events. The CR group tended to peak on day 2, whereas the WC sheep levels

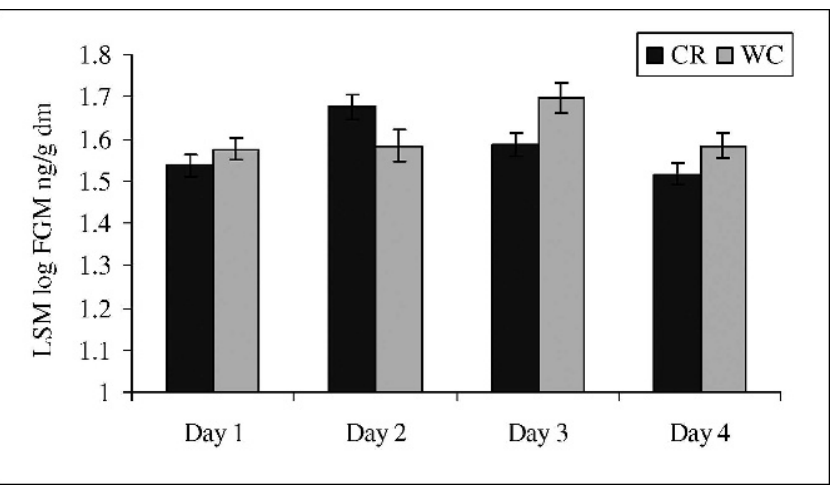

Figure 5. Changes in fecal glucocorticoid metabolites (FGM) in bighorn sheep in response to handling at the Animal Population Health Institute, Colorado State University animal facility, Fort Collins, Colorado, USA, February-July 2004. Base-10 log-transformed least squares means (LSM) for FGM in captive-raised (CR) and wild-caught (WC) bighorn sheep for the day of and the 3 days following handling events are shown. We combined the results from 8 sampling events over 6 months.

peaked on day 3 (Fig. 5). The LSM of log-transformed FGM levels for CR sheep were significantly higher than those for WC sheep on day 2 (CR LSM $=1.68$, WC LSM $=1.58, P=0.048, \mathrm{df}=156)$, and LSM of WC levels were significantly higher than those for CR on day 3 (CR LSM $=1.59, \mathrm{WC} \mathrm{LSM}=1.70, P=0.014, \mathrm{df}=156)$. Mean differences between the 2 groups on days 1 (CR LSM = 1.54 , WC LSM $=1.58, P=0.28)$ and $4(\mathrm{CR} \mathrm{LSM}=1.52$, $\mathrm{WC} \operatorname{LSM}=1.58, P=0.10, \mathrm{df}=156)$ were not statistically significant. For both groups, there was no significant difference in mean FGM levels between collection days 1 and $4(P=0.54$ for $\mathrm{CR}$, and $P=0.83$ for $\mathrm{WC})$, indicating a return to baseline levels within 3 days following handling events.

\section{DISCUSSION}

Our objectives were to compare the endocrine responses to an acute stress event between 2 groups of sheep and to compare changes in baseline FGM levels over time. We found that although the WC and CR sheep had very similar HPA responses to an acute stress event, the epinephrine levels for the CR sheep were consistently higher than those for the WC sheep. The general trend of the higher epinephrine levels in CR versus WC sheep, in conjunction with very similar plasma cortisol levels during the drop-net between the 2 groups, may be explained by the distinction between the HPA and ANS responses. Due to previous experiences in captivity and human contact, the CR sheep may have perceived that they still had some control over the situation, as compared to the WC sheep. The differences that we found between the 2 groups of sheep in the ANS and HPA response indicate that several measurements, rather than measurement of a single hormone or endocrine system, may be necessary to characterize different components of the stress response and to determine when normal adaptive stress has crossed the threshold and may lead to decreased fitness.

The monthly handling events served as an acute stress event repeated over time. The overall decreasing trend in 
day 1 fecal cortisol levels for both groups was likely due to a combination of the animals becoming progressively more acclimated to captivity and handling and by the human personnel becoming more efficient at handling and collecting samples. Decreasing trends in baseline cortisol levels have been used in other species to confirm that a change in environmental conditions in captivity had decreased stress (Carlsted and Shepherdson 2000). Visual barriers, a predictable routine, and contact with tame hybrid sheep have all been shown to help wild sheep acclimate more quickly, as determined by behavioral observations (Boyce et al. 1992).

Our results emphasize the importance of long-term monitoring and establishing average baseline levels for a given individual or herd for valid interpretation of stress hormone measurements (Morrow et al. 2002, Millspaugh and Washburn 2004). When using neuroendocrine measures of the stress response as an indication of animal wellbeing, it is generally chronic stress, rather than acute stress events, that correlates to detrimental biological effects (Cyr and Romero 2007). Because cortisol metabolites are pooled over a longer period of time in fecal samples as compared to plasma samples, it helps to eliminate the problem of confounding the measurement with the method of handling. However, these hormones fluctuate daily. Long-term periodic monitoring may help differentiate between a shortterm adaptive and appropriate stress response to a particular situation versus a chronic stress response, which may be due to human disturbances, predatory stress, or high population density.

Our sampling design of repeated collection days within repeated sampling periods was established to capture changes in both the baseline FGM levels and the response to handling over time. We assumed that the FGM levels on the day the animals were handled were baseline because a given fecal sample represents cortisol production approximately 12 hours earlier, and there is no indication that any unique stressors were present in the days prior to handling (Möstl et al. 2002). By collecting samples for 3 additional days and observing the rise in FGM levels and a return to day 1 FGM levels, we were able to lend credibility to this assumption.

We were able to detect an overall downward trend in baseline FGM levels, with the exception of the increase at sample period 5. It is noteworthy that the HPA stress responses at period 5 of both groups were similar, even though we were unable to identify the specific cause of the increase. Despite the different backgrounds and experience in captivity prior to our study, after only 5 weeks, the endocrine response of the 2 groups was the same.

Good techniques and assays have been developed to measure neuroendocrine hormone levels, but the results must be interpreted within the context of the wide range of factors that influence a given hormone level at a given point in time. The general management goal of measuring neuroendocrine hormones is to make an educated assessment regarding the stress that a particular animal or herd is experiencing as it relates to animal well-being. The difficulty in both human and animal research is determining when the threshold has been crossed between a normal and adaptive response to stress and a response that is of a sufficient magnitude or duration to be biologically detrimental and cause negative impacts to metabolism, growth, immune function, or reproduction. We did not see any clinical signs indicating that the threshold levels had been crossed. However, we were unable to use quantitative measures of biological endpoints, such as reproductive success or the strength of the immune response, to determine if any correlation existed between the hormones levels we measured and these endpoints. Further confounding the assessment of threshold is that it may be different from one animal to another. A statement of well-being of a particular animal cannot automatically be obtained from a specific hormone level, rather an overall trend should be assessed (Millspaugh and Washburn 2004). More information concerning the levels of glucocorticoids, or other endocrine parameters, that specifically correlate to a decrease in fitness is needed through additional research with a variety of animal species. Furthermore, other studies have found high degrees of both intra- and inter-species variability in normal cortisol levels. We were unable to statistically analyze variables that play a role in individual animal glucocorticoid levels including genetics, age, sex, gestational stage, and early life experiences (Millspaugh and Washburn 2004, Palme 2005, Lane 2006). Therefore, it is difficult to attribute changes in glucocorticoid levels to a specific stressor. Future stress studies with bighorn sheep should seek to address some of these issues to further improve the interpretation and utility of stress hormone data and to be able to effectively apply these techniques to translocation and reintroduction efforts.

\section{MANAGEMENT IMPLICATIONS}

Fecal glucocorticoid metabolites provide a noninvasive mechanism to monitor one component of the stress response in wild and captive bighorn sheep populations. Managers could use FGM as one more parameter to document as animals are periodically handled for other management purposes or studies. If baseline levels for a given bighorn sheep herd are known, then changes or long-term increases in these levels may be used to assess herd health as it correlates to the expansion of roads and recreation sites, mining and oil extraction, snowmobiles in winter and offroad vehicles in the summer, and domestic grazing contracts and sheep-hunting quotas. If a significant increase, such as we observed, occurs as an isolated event, there would be less likelihood of long-term consequences. However, if FGM levels have been stable at baseline levels and then an increase occurs without a subsequent decrease, suggesting chronic stress, there may be more risk of changes in other biological functions. If some stressor could be identified in correlation with that increase, then perhaps there may be an opportunity for managers to intervene at this time. In the context of wild sheep in captivity, FGM can be especially helpful for monitoring baseline stress during the acclimation process for new animals, thus providing an opportunity to 
change the social dynamics, housing, or enrichment available to the animals to help decrease physiologic stress and improve overall health.

\section{ACKNOWLEDGMENTS}

We extend gratitude to the many people that contributed time, expertise, and hard work to the completion of this project. The Animal Population Health Institute, Colorado State University, and the Foundation for North American Wild Sheep provided funding. P. Nol and K. Held at The United States Department of Agriculture, Animal and Plant Health Inspection Service (USDA APHIS) and R. Thompson and D. Miller at Colorado State University assisted with all aspects of animal handling, sampling, and sample processing. K. Crane, USDA APHIS, conducted radioimmunoassays. J. George and M. Vierra, as well as many other Colorado Division of Wildlife personnel, assisted with equipment and sampling. Many others at the National Wildlife Research Center and the Center for Epidemiology and Animal Health assisted with sample collection.

\section{LITERATURE CITED}

Arlettaz, R., P. Patthey, M. Baltic, T. Leu, M. Schaub, R. Palme, and S. Jenni-Eiermann. 2007. Spreading free-riding snow sports represent a novel serious threat for wildlife. Proceedings of the Royal Society Biological Sciences 274:1219-1224.

Bailey, J. A., and D. R. Klein. 1997. United States of America. Pages 307316 in D. M. Shackleton, editor. Wild sheep and goats and their relatives: status survey and conservation action plan for Caprinae. International Union for Conservation of Nature and Natural Resources, Gland, Switzerland.

Belden, E. L., E. S. Williams, E. T. Thorne, H. J. Harlow, K. White, and S. L. Anderson. 1990. Effect of chronic stress on immune system function of Rocky Mountain bighorn sheep. Proceedings of the Biennial Symposium of the Northern Wild Sheep and Goat Council 7:76-91.

Boyce, W., T. Bunch, J. R. DeForge, D. A. Jessup, and J. D. Wehausen. 1992. Panel discussions: stress and bighorn sheep. Transactions of the Desert Bighorn Council 36:58-68.

Buechner, H. K., 1960. The bighorn sheep in the United States, its past, present, and future. Wildlife Monographs 4.

Carlsted, K., and D. Shepherdson. 2000. Alleviating stress in zoo animals with environmental enrichment. Pages 337-354 in G. P. Moberg and J. A. Mench, editors. The biology of animal stress. CABI, New York, New York, USA.

Corley, K. C., H. P. Mauck, and F. O. M. Shiel. 1975. Cardiac responses associated with "yoked-chain" shock avoidance in squirrel monkeys. Psychophysiology 12:439-444.

Creel, S., N. M. Creel, and S. L. Monfort. 1997. Radiocollaring and stress hormones in African wild dogs. Conservation Biology 11:544-548.

Creel, S., J. E. Fox, A. Hardy, J. Sands, B. Garrott, and R. O. Peterson. 2002. Snowmobile activity and glucocorticoid stress responses in wolves and elk. Conservation Biology 16:809-814.

Cyr, N. E., and M. Romero. 2007. Chronic stress in free-living European starlings reduces corticosterone concentrations and reproductive success. General and Comparative Endocrinology 151:82-89.

de Boer, S. F., R. de beun, J. L. Slangen, and J. van der Gugten. 1990. Dynamics of plasma catecholamine and corticosterone concentrations during reinforced and extinguished behavior in rats. Physiology \& Behavior 47:691-698.

de Kloet, E. R. 2004. Hormones and the stressed brain. Annals of the New York Academy of Sciences 1018:1-15.

Festa-Bianchet, M. 1988. A pneumonia epizootic in bighorn sheep, with comments on preventative management. Proceedings of the Biennial Symposium of the Northern Wild Sheep and Goat Council 6:66-76.
Forrester, D. J. 1971. Bighorn sheep lungworm-pneumonia complex. Pages 158-173 in J. W. Davis and R. C. Anderson, editors. Parasitic diseases of wild mammals. Iowa State Press, Ames, USA.

Henry, J. P. 1993. Biological basis of the stress response. Neural Information Processing Systems 8:69-73.

Ladewig, J. 2000. Chronic intermittent stress: a model for the study of long-term stressors. Pages 159-169 in G. P. Moberg and J. A. Mench, editors. The biology of animal stress. CABI, New York, New York, USA.

Lane, J. 2006. Can non-invasive glucocorticoid measures be used as reliable indicators of stress in animals? Animal Welfare 15:331-342.

Li, C., Z. Jiang, S. Tang, and Y. Zeng. 2007. Influence of enclosure size and animal density on fecal cortisol concentration and aggression in Pere David's deer stags. General and Comparative Endocrinology 151:202209.

Miller, M. W., N. T. Hobbs, and M. C. Sousa. 1991. Detecting stress responses in Rocky Mountain bighorn sheep (Ovis canadensis canadensis): reliability of cortisol concentrations in urine and feces. Canadian Journal of Zoology 69:15-24.

Millspaugh, J. J., and B. E. Washburn. 2004. Use of fecal glucocorticoid metabolite measures in conservation biology research: considerations for application and interpretation. General and Comparative Endocrinology 138:189-199.

Millspaugh, J. J., B. E. Washburn, M. A. Milanick, J. Beringer, L. P. Hansen, and T. M. Meyer. 2002. Non-invasive techniques for stress assessment in white-tailed deer. Wildlife Society Bulletin 30:899-907.

Millspaugh, J. J., R. J. Woods, K. E. Hunt, G. C. Raedeke, B. E. Washburn, and S. K. Wasser. 2001. Fecal glucocorticoid assays and the physiological stress response in elk. Wildlife Society Bulletin 29:899-907.

Moberg, G. P. 2000. Biological response to stress: implications for animal welfare. Pages 1-21 in G. P. Moberg and J. A. Mench, editors. The biology of animal stress. CABI, New York, New York, USA.

Monello, R. J., D. L. Murray, and E. F. Cassirer. 2001. Ecological correlates of pneumonia epizootics in bighorn sheep herds. Canadian Journal of Zoology 79:1423-1432.

Morrow, C. J., E. S. Kolver, G. A. Verkerk, and L. R. Matthews. 2002. Fecal glucocorticoid metabolites as a measure of adrenal activity in dairy cattle. General and Comparative Endocrinology 126:229-241.

Möstl, E., J. L. Maggs, G. Schrotter, U. Besenfelder, and R. Palme. 2002. Measurement of cortisol metabolites in faeces of ruminants. Veterinary Research Communications 26:127-139.

Palme, R. 2005. Measuring fecal steroids guidelines for practical application. Annals of the New York Academy of Sciences 1046:1-6.

Palme, R., C. Robia, S. Messmann, J. Hofer, and E. Möstl. 1999. Measurement of faecal cortisol metabolites in ruminants: a non-invasive parameter of adrenocortical function. Wiener Tierarztliche Monatsschrift 86:237-241.

Seligman, M. E. P., and S. F. Maier. 1967. Failure to escape traumatic shock. Journal of Experimental Psychology 74:1-9.

Silflow, R. M., W. J. Foreyt, S. M. Taylor, W. W. Laegreid, H. D. Liggitt, and R. W. Leid. 1989. Comparison of pulmonary defense mechanisms in Rocky Mountain bighorn (Ovis canadensis canadensis) and domestic sheep. Journal of Wildlife Diseases 25:514-520.

Singer, F. J., and M. A. Gudorf. 1999. Restoration of bighorn sheep metapopulations in and near 15 national parks: conservation of a severely fragmented species. U.S. Geological Survey, Report no. 99-102, Fort Collins, Colorado, USA.

Spraker, T. R., C. P. Hibler, G. G. Schoonveld, and W. S. Adney. 1984. Pathologic changes and microorganisms found in bighorn sheep during a stress-related die-off. Journal of Wildlife Diseases 20:319-327.

Teixeira, C., C. Schetini de Azevedo, M. Mendl, C. F. Cipreste, and R. J. Young. 2007. Revisiting translocation and reintroduction programmes: the importance of considering stress. Animal Behaviour 73:1-13.

Ullrey, D. E. 1993. Nutrition and predisposition to infectious disease. Journal of Zoo and Wildlife Medicine 24:304-314.

Wasser, S. K., K. E. Hunt, J. L. Brown, K. Cooper, C. M. Crockett, U. Bechert, J. J. Millspaugh, S. Larson, and S. L. Monfort. 2000. A generalized fecal glucocorticoid assay for use in a diverse array of nondomestic mammalian and avian species. General and Comparative Endocrinology 120:260-275.

Associate Editor: Hudson. 